\title{
Recursos para la salud en unidades de la Secretaría de Salud, 1999
}

U

no de los principales objetivos de la Secretaría de Salud (SSA), es otorgar atención a la salud de la población que no goza del servicio de seguridad social, para lo cual, cuenta con los recursos humanos, materiales y físicos, organizados de tal forma que puedan brindar un adecuado servicio a la comunidad que así lo requiere.

El Subsistema de Recursos forma parte del Sistema de Información en Salud para Población Abierta (SISPA) de la SSA, lo que permite conocer los recursos con que cuenta la propia SSA para brindar atención a la población demandante del servicio. Junto con los otros tres subsistemas: Población, Prestación de Servicios y Daños a la Salud, la información forma parte de las estadísticas del sector salud.
Por otra parte, con el Registro Nacional de Infraestructura para la Salud (RENIS), que se obtiene por medio de una cédula anual que llenan los responsables de cada unidad aplicativa, referente a recursos humanos, materiales y físicos con que cuenta ésta para proporcionar atención preventiva, curativa y, en su caso, de rehabilitación, se conoce la ubicación física, el tipo de unidad y el personal disponible en la misma unidad.

Asimismo, la Dirección General de Estadística e Informática tiene, entre sus obligaciones, dar a conocer la información al 31 de diciembre de cada año, respecto de los recursos de la Secretaría de Salud.

A continuación se presenta la información referente a los recursos de las unidades médicas de la Se- cretaría de Salud con que contaron durante 1999. En esta información se incluyen variables elegidas de los diferentes recursos dada su importancia para la prestación de los servicios de salud. Los datos son por entidad federativa y tipo de unidad, y éstos aparecen en números absolutos y relativos.

Para los usuarios existe información disponible con mayor desagregación en el Web de la SSA y en las diversas publicaciones de la Dirección General de Estadística e Informática (sita en Leibnitz 20, 3er. piso, colonia Nueva Anzures, 11590 México, D.F., México) como son: el Anuario Estadístico de la Secretaría de Salud, el Boletín de Información Estadística Sectorial, y el Inventario de Recursos Humanos y Materiales. 


\section{Recursos materiales en las unidades médicas. Secretaría de Salud, México, 1999}

Entidad federativa

\begin{tabular}{|c|c|c|c|c|c|c|c|c|c|c|c|c|c|}
\hline \multicolumn{3}{|c|}{ Unidades médicas } & \multirow[b]{2}{*}{ Con- } & \multirow{2}{*}{\multicolumn{2}{|c|}{ Camas }} & \multirow[b]{2}{*}{ Incu- } & \multirow[b]{3}{*}{$\begin{array}{l}\text { Quiró- } \\
\text { fanos }\end{array}$} & \multirow{3}{*}{$\begin{array}{l}\text { Salas } \\
\text { de } \\
\text { expulsión }\end{array}$} & \multicolumn{3}{|c|}{ Laboratorios } & \multirow{2}{*}{\multicolumn{2}{|c|}{$\frac{\text { Equipos }}{\text { Electro- }}$}} \\
\hline & & Hospitalización & & & & & & & Análi- & & Ultra- & & \\
\hline Total & $\begin{array}{l}\text { Consulta } \\
\text { externa }\end{array}$ & $\begin{array}{l}\text { Gene- Especia- } \\
\text { ral lizada }\end{array}$ & $\begin{array}{l}\text { sulto- } \\
\text { rios }\end{array}$ & $\begin{array}{l}\text { Censa- } \\
\text { bles }\end{array}$ & $\begin{array}{c}\text { No } \\
\text { censables }\end{array}$ & $\begin{array}{c}\text { bado- } \\
\text { ras }\end{array}$ & & & $\begin{array}{l}\text { sis } \\
\text { cínicos }\end{array}$ & $\begin{array}{l}\text { Pato- } \\
\text { logía }\end{array}$ & $\begin{array}{l}\text { sono- } \\
\text { grafía }\end{array}$ & $\begin{array}{c}\text { Rayos } \\
X\end{array}$ & $\begin{array}{l}\text { cardio- } \\
\text { grafía }\end{array}$ \\
\hline
\end{tabular}

Estados Unidos Mexicanos $1049310088 \quad 310 \quad 95 \quad 22202 \quad 31252 \quad 31986 \quad 2361 \quad 1085 \quad 5708 \quad 893 \quad 120 \quad 389 \quad 1107 \quad 842 \quad 3396$

\begin{tabular}{|c|c|c|c|c|c|c|c|c|c|c|c|c|c|c|c|c|}
\hline A guascalientes & 84 & 77 & 4 & 3 & 249 & 394 & 334 & 33 & 14 & 52 & 6 & 1 & 6 & 18 & 17 & 40 \\
\hline Baja California & 113 & 110 & 3 & 0 & 307 & 365 & 168 & 36 & 13 & 37 & 5 & 3 & 3 & 10 & 16 & 49 \\
\hline Baja California Sur & 75 & 70 & 3 & 2 & 140 & 220 & 201 & 20 & 10 & 35 & 7 & 2 & 3 & 15 & 12 & 14 \\
\hline Campeche & 144 & 133 & 9 & 2 & 281 & 352 & 352 & 33 & 15 & 46 & 12 & 2 & 6 & 15 & 16 & 24 \\
\hline Coahuila & 182 & 169 & 10 & 3 & 429 & 465 & 442 & 37 & 20 & 57 & 20 & 0 & 11 & 27 & 15 & 89 \\
\hline Colima & 116 & 112 & 4 & 0 & 219 & 201 & 392 & 19 & 7 & 59 & 7 & 1 & 3 & 9 & 7 & 24 \\
\hline Chiapas & 328 & 314 & 14 & 0 & 795 & 724 & 824 & 63 & 41 & 149 & 40 & 1 & 8 & 39 & 15 & 148 \\
\hline Chihuahua & 265 & 247 & 14 & 4 & 463 & 838 & 716 & 70 & 29 & 125 & 27 & 4 & 12 & 29 & 22 & 47 \\
\hline Distrito Federal & 368 & 326 & 11 & 31 & 2523 & 4977 & 1588 & 408 & 181 & 40 & 118 & 20 & 62 & 199 & 124 & 454 \\
\hline Durango & 186 & 178 & 7 & 1 & 353 & 490 & 844 & 41 & 18 & 103 & 23 & 2 & 5 & 17 & 12 & 33 \\
\hline Guanajuato & 491 & 478 & 11 & 2 & 888 & 988 & 1856 & 81 & 30 & 378 & 29 & 4 & 14 & 54 & 24 & 81 \\
\hline Guerrero & 878 & 864 & 14 & 0 & 1254 & 830 & 2156 & 77 & 40 & 613 & 54 & 2 & 15 & 46 & 24 & 111 \\
\hline Hidalgo & 405 & 397 & 7 & 1 & 618 & 720 & 1352 & 61 & 17 & 359 & 27 & 1 & 9 & 16 & 14 & 78 \\
\hline Jalisco & 717 & 700 & 13 & 4 & 1444 & 2463 & 2779 & 156 & 99 & 477 & 47 & 6 & 26 & 55 & 39 & 202 \\
\hline México & 971 & 937 & 29 & 5 & 2290 & 4285 & 3038 & 246 & 82 & 369 & 69 & 12 & 26 & 89 & 100 & 523 \\
\hline Michoacán & 395 & 380 & 12 & 3 & 690 & 884 & 1567 & 74 & 35 & 209 & 31 & 3 & 13 & 45 & 24 & 120 \\
\hline Morelos & 212 & 206 & 5 & 1 & 427 & 340 & 698 & 32 & 12 & 115 & 11 & 3 & 3 & 15 & 15 & 57 \\
\hline $\mathrm{N}$ ayarit & 195 & 191 & 4 & 0 & 298 & 308 & 472 & 22 & 15 & 100 & 13 & 1 & 2 & 14 & 9 & 40 \\
\hline Nuevo León & 443 & 434 & 6 & 3 & 845 & 625 & 637 & 36 & 22 & 43 & 35 & 2 & 18 & 25 & 12 & 147 \\
\hline 0 axaca & 576 & 562 & 13 & 1 & 713 & 774 & 1134 & 58 & 24 & 396 & 22 & 2 & 12 & 23 & 28 & 77 \\
\hline Puebla & 471 & 448 & 22 & 1 & 925 & 1201 & 1614 & 78 & 34 & 343 & 26 & 3 & 17 & 44 & 37 & 235 \\
\hline Q uerétaro & 236 & 231 & 4 & 1 & 336 & 374 & 722 & 38 & 13 & 142 & 10 & 2 & 8 & 16 & 10 & 48 \\
\hline Q uintana Roo & 166 & 161 & 4 & 1 & 279 & 248 & 356 & 29 & 14 & 85 & 20 & 1 & 2 & 10 & 8 & 29 \\
\hline San Luis Potosí & 259 & 253 & 4 & 2 & 377 & 597 & 610 & 65 & 18 & 111 & 13 & 3 & 6 & 11 & 14 & 101 \\
\hline Sinaloa & 207 & 199 & 7 & 1 & 409 & 480 & 763 & 27 & 22 & 154 & 14 & 3 & 9 & 19 & 17 & 46 \\
\hline Sonora & 228 & 210 & 15 & 3 & 561 & 1022 & 978 & 56 & 32 & 178 & 25 & 3 & 6 & 39 & 27 & 51 \\
\hline Tabasco & 503 & 488 & 13 & 2 & 824 & 820 & 1279 & 50 & 35 & 273 & 38 & 3 & 10 & 25 & 23 & 176 \\
\hline Tamaulipas & 245 & 231 & 12 & 2 & 540 & 1122 & 690 & 80 & 35 & 87 & 24 & 7 & 13 & 30 & 23 & 51 \\
\hline Tlaxcala & 168 & 163 & 4 & 1 & 308 & 253 & 596 & 26 & 11 & 144 & 8 & 1 & 4 & 12 & 8 & 45 \\
\hline Veracruz & 543 & 513 & 26 & 4 & 1192 & 1627 & 1382 & 110 & 73 & 231 & 43 & 7 & 19 & 45 & 36 & 165 \\
\hline Yucatán & 155 & 150 & 3 & 2 & 311 & 488 & 432 & 25 & 14 & 68 & 9 & 2 & 3 & 10 & 11 & 31 \\
\hline Zacatecas & 159 & 156 & 3 & 0 & 265 & 288 & 559 & 51 & 18 & 126 & 17 & 2 & 3 & 10 & 7 & 31 \\
\hline Institutos nacionales & 9 & 0 & 0 & 9 & 649 & 1489 & 455 & 123 & 42 & 4 & 43 & 11 & 32 & 76 & 76 & 29 \\
\hline
\end{tabular}

Fuente: Secretaría de Salud (SSA), Subsecretaría de Prevención y Control de Enfermedades, Dirección General de Estadística e Informática (D G El), Sistema de Información en Salud para Población A bierta (SISPA), Registro N acional de Infraestructura para la Salud (REN IS) 


\section{Recursos físicos y materiales por tipo de unidad. Secretaría de Salud, México, 1999}

\begin{tabular}{|c|c|c|c|c|c|c|c|c|c|c|c|c|c|}
\hline \multirow{3}{*}{ Tipo de unidad } & \multirow{3}{*}{$\begin{array}{l}\text { Unidades } \\
\text { médicas }\end{array}$} & \multirow{3}{*}{$\begin{array}{c}\text { Consul- } \\
\text { torios }\end{array}$} & \multirow{2}{*}{\multicolumn{2}{|c|}{ Camas }} & \multirow{3}{*}{$\begin{array}{c}\text { Incuba- } \\
\text { doras }\end{array}$} & \multirow{3}{*}{$\begin{array}{l}\text { Quiró- } \\
\text { fano }\end{array}$} & \multirow{3}{*}{$\begin{array}{l}\text { Salas de } \\
\text { expulsión }\end{array}$} & \multicolumn{3}{|c|}{ Laboratorios } & \multicolumn{3}{|c|}{ Equipos } \\
\hline & & & & & & & & Análisis & Pato- & Ultraso- & Rayos & Electrocar- & Unidad \\
\hline & & & Censables & No censables & & & & clínicos & logía & nografía & $x$ & diografía & dental \\
\hline Estados Unidos Mexicanos & 10493 & 22202 & 31252 & 31986 & 2361 & 1085 & 5708 & 893 & 120 & 389 & 1107 & 842 & 3396 \\
\hline Unidad rural & 7033 & 8803 & 1 & 19653 & 5 & 1 & 4941 & 61 & 1 & 0 & 41 & 3 & 862 \\
\hline Unidad móvil & 1410 & 796 & 0 & 90 & 2 & 0 & 2 & 0 & 0 & 0 & 0 & 0 & 380 \\
\hline Unidad urbana & 1422 & 5571 & 12 & 1135 & 0 & 4 & 150 & 255 & 0 & 2 & 133 & 3 & 1347 \\
\hline Clínica de especialidad & 63 & 412 & 24 & 195 & 2 & 16 & 1 & 15 & 2 & 5 & 18 & 4 & 147 \\
\hline C. de S. con hospitalización & 160 & 858 & 1637 & 825 & 166 & 128 & 160 & 128 & 0 & 5 & 92 & 25 & 168 \\
\hline Hospital integral & 34 & 219 & 440 & 253 & 59 & 35 & 34 & 32 & 0 & 5 & 37 & 19 & 43 \\
\hline Hospital general & 276 & 3227 & 16210 & 7062 & 1444 & 606 & 360 & 274 & 79 & 254 & 518 & 548 & 296 \\
\hline Hospital especializado & 56 & 1201 & 6238 & 2021 & 560 & 252 & 56 & 69 & 25 & 86 & 183 & 148 & 99 \\
\hline Hospital psiquiátrico & 30 & 466 & 5201 & 297 & 0 & 1 & 0 & 16 & 2 & 0 & 9 & 16 & 25 \\
\hline Institutos nacionales & 9 & 649 & 1489 & 455 & 123 & 42 & 4 & 43 & 11 & 32 & 76 & 76 & 29 \\
\hline
\end{tabular}

Fuente: Secretaría de Salud (SSA), Subsecretaría de Prevención y Control de Enfermedades, D irección G eneral de Estadística e Informática (D G El), Sistema de Información en Salud para Población A bierta (SISPA), Registro N acional de Infraestructura para la Salud (REN IS) 


\section{Cuadro III (Primera parte) \\ Recursos humanos en las unidades médicas. Secretaría de Salud, México, 1999}

\begin{tabular}{|c|c|c|c|c|c|c|c|c|c|c|c|c|c|}
\hline \multirow[b]{2}{*}{ Entidad federativa } & \multirow[b]{2}{*}{$\begin{array}{l}\text { M édicos en } \\
\text { contacto }\end{array}$} & \multirow[b]{2}{*}{$\begin{array}{c}\text { Otra } \\
\text { actividad }\end{array}$} & \multicolumn{7}{|c|}{ M édicos en contacto con el paciente } & \multicolumn{4}{|c|}{ En formación } \\
\hline & & & $\begin{array}{l}\text { General y } \\
\text { familiar }\end{array}$ & $\begin{array}{l}\text { Pedia- } \\
\text { tra }\end{array}$ & $\begin{array}{l}\text { Gineco- } \\
\text { obstetra }\end{array}$ & $\begin{array}{l}\text { Ciru- } \\
\text { jano }\end{array}$ & $\begin{array}{l}\text { Inter- } \\
\text { nista }\end{array}$ & $\begin{array}{c}\text { Otra } \\
\text { especialidad }\end{array}$ & $\begin{array}{l}\text { Odon- } \\
\text { tólogo }\end{array}$ & $\begin{array}{l}\text { Pasante } \\
\text { medicina }\end{array}$ & $\begin{array}{c}\text { Pasante } \\
\text { odontólogo }\end{array}$ & $\begin{array}{l}\text { Interno de } \\
\text { pregrado }\end{array}$ & $\begin{array}{l}\text { Resi- } \\
\text { dentes }\end{array}$ \\
\hline Estados Unidos Mexicanos & $5 \quad 46057$ & 4252 & 13239 & 2200 & 2073 & 1985 & 1088 & 6769 & 3238 & 5840 & 1609 & 3254 & 4762 \\
\hline A guascalientes & 778 & 60 & 156 & 55 & 40 & 44 & 24 & 159 & 27 & 59 & 26 & 100 & 88 \\
\hline Baja California & 699 & 72 & 143 & 33 & 35 & 33 & 22 & 94 & 42 & 64 & 50 & 77 & 106 \\
\hline Baja California Sur & 339 & 18 & 83 & 16 & 20 & 21 & 13 & 63 & 14 & 57 & 3 & 26 & 23 \\
\hline Campeche & 490 & 23 & 177 & 24 & 34 & 27 & 15 & 64 & 25 & 61 & 12 & 35 & 16 \\
\hline Coahuila & 764 & 73 & 229 & 40 & 53 & 36 & 25 & 128 & 44 & 95 & 63 & 35 & 16 \\
\hline Colima & 392 & 24 & 100 & 16 & 21 & 16 & 13 & 62 & 22 & 76 & 11 & 28 & 27 \\
\hline Chiapas & 1307 & 96 & 529 & 52 & 60 & 76 & 25 & 146 & 169 & 112 & 14 & 95 & 29 \\
\hline Chihuahua & 1005 & 61 & 364 & 58 & 49 & 61 & 32 & 162 & 52 & 86 & 10 & 52 & 79 \\
\hline Distrito Federal & 6541 & 956 & 1509 & 426 & 357 & 298 & 132 & 1690 & 662 & 73 & 166 & 259 & 969 \\
\hline Durango & 747 & 53 & 242 & 43 & 33 & 38 & 23 & 118 & 50 & 77 & 14 & 49 & 60 \\
\hline Guanajuato & 1662 & 149 & 684 & 54 & 55 & 51 & 30 & 128 & 73 & 318 & 73 & 115 & 81 \\
\hline Guerrero & 1942 & 88 & 916 & 68 & 83 & 73 & 31 & 154 & 122 & 323 & 12 & 132 & 28 \\
\hline Hidalgo & 1103 & 159 & 425 & 21 & 23 & 34 & 19 & 76 & 93 & 259 & 13 & 84 & 56 \\
\hline Jalisco & 3691 & 301 & 651 & 126 & 161 & 141 & 89 & 535 & 177 & 595 & 153 & 460 & 603 \\
\hline México & 4056 & 427 & 1163 & 151 & 178 & 162 & 128 & 338 & 365 & 668 & 207 & 339 & 357 \\
\hline Michoacán & 1572 & 97 & 412 & 43 & 65 & 58 & 38 & 199 & 93 & 289 & 128 & 171 & 76 \\
\hline Morelos & 809 & 48 & 199 & 59 & 47 & 41 & 27 & 148 & 53 & 153 & 29 & 37 & 16 \\
\hline$N$ ayarit & 564 & 33 & 165 & 21 & 21 & 26 & 15 & 48 & 37 & 126 & 26 & 49 & 30 \\
\hline Nuevo León & 1280 & 96 & 383 & 60 & 62 & 30 & 25 & 114 & 71 & 276 & 90 & 54 & 115 \\
\hline 0 axaca & 1400 & 73 & 536 & 31 & 31 & 50 & 24 & 133 & 61 & 247 & 99 & 134 & 54 \\
\hline Puebla & 1627 & 82 & 481 & 41 & 44 & 65 & 24 & 138 & 119 & 335 & 157 & 135 & 88 \\
\hline Q uerétaro & 815 & 52 & 197 & 57 & 50 & 30 & 14 & 88 & 46 & 185 & 17 & 72 & 59 \\
\hline Q uintana Roo & 507 & 34 & 228 & 25 & 26 & 29 & 12 & 45 & 38 & 55 & 6 & 27 & 16 \\
\hline San Luis Potosí & 933 & 46 & 256 & 40 & 48 & 26 & 13 & 120 & 93 & 93 & 29 & 90 & 125 \\
\hline Sinaloa & 906 & 86 & 258 & 50 & 48 & 51 & 27 & 156 & 49 & 124 & 21 & 60 & 62 \\
\hline Sonora & 1082 & 107 & 218 & 59 & 49 & 49 & 25 & 192 & 59 & 154 & 5 & 97 & 175 \\
\hline Tabasco & 1758 & 108 & 650 & 55 & 66 & 71 & 28 & 226 & 206 & 266 & 20 & 59 & 111 \\
\hline Tamaulipas & 1315 & 112 & 376 & 88 & 85 & 70 & 60 & 212 & 55 & 109 & 39 & 100 & 121 \\
\hline Tlaxcala & 550 & 22 & 183 & 24 & 20 & 30 & 8 & 51 & 44 & 117 & 15 & 31 & 27 \\
\hline Veracruz & 2308 & 131 & 943 & 79 & 101 & 126 & 65 & 300 & 151 & 197 & 85 & 148 & 113 \\
\hline Yucatán & 730 & 67 & 217 & 49 & 38 & 24 & 25 & 112 & 35 & 89 & 8 & 59 & 74 \\
\hline Zacatecas & 546 & 33 & 158 & 26 & 31 & 28 & 17 & 61 & 55 & 102 & 8 & 45 & 15 \\
\hline Institutos nacionales & 1839 & 465 & 8 & 210 & 39 & 70 & 20 & 509 & 36 & 0 & 0 & 0 & 947 \\
\hline
\end{tabular}

Fuente: Secretaría de Salud (SSA), Subsecretaría de Prevención y Control de Enfermedades, Dirección General de Estadística e Informática (D G El), Sistema de Información en Salud para Población Abierta (SISPA), Registro N acional de Infraestructura para la Salud (REN IS) 
Cuadro III (Segunda parte)

Recursos humanos en las unidades médicas. Secretaría de Salud, México, 1999

\begin{tabular}{|c|c|c|c|c|c|c|c|c|c|c|c|c|c|}
\hline \multirow[b]{2}{*}{ Entidad federativa } & \multicolumn{6}{|c|}{ Enfermeras en contacto con el paciente } & \multicolumn{7}{|c|}{ Otro personal } \\
\hline & Total & $\begin{array}{c}\text { Gene- } \\
\text { ral }\end{array}$ & $\begin{array}{l}\text { Especia- } \\
\text { lista }\end{array}$ & $\begin{array}{l}\text { Pa- } \\
\text { sante }\end{array}$ & $\begin{array}{l}\text { Auxi- } \\
\text { liar }\end{array}$ & $\begin{array}{l}\text { Otra } \\
\text { labor }\end{array}$ & Total & $\begin{array}{l}\text { Quí- } \\
\text { mico }\end{array}$ & $\begin{array}{c}\text { Trabajo } \\
\text { social }\end{array}$ & $\begin{array}{c}\text { Técnico } \\
\text { laboratorio }\end{array}$ & Radiólogo & $\begin{array}{l}\text { Citotec- } \\
\text { nólogo }\end{array}$ & Otro \\
\hline Estados Unidos Mexicanos & 64797 & 30078 & 3820 & 8192 & 22707 & 4881 & 73410 & 2251 & 4132 & 3293 & 1812 & 239 & 61683 \\
\hline Aguascalientes & 959 & 328 & 66 & 79 & 486 & 43 & 999 & 18 & 49 & 43 & 34 & 1 & 854 \\
\hline Baja California & 1052 & 485 & 63 & 100 & 404 & 143 & 921 & 24 & 69 & 42 & 28 & 4 & 754 \\
\hline Baja California Sur & 463 & 312 & 4 & 34 & 113 & 20 & 622 & 29 & 40 & 21 & 23 & 3 & 506 \\
\hline Campeche & 612 & 258 & 16 & 48 & 290 & 38 & 617 & 27 & 30 & 28 & 20 & 3 & 509 \\
\hline Coahuila & 1206 & 653 & 108 & 364 & 81 & 78 & 984 & 41 & 58 & 25 & 30 & 8 & 822 \\
\hline Colima & 488 & 341 & 23 & 49 & 75 & 26 & 444 & 14 & 23 & 31 & 16 & 4 & 356 \\
\hline Chiapas & 1960 & 695 & 23 & 248 & 994 & 76 & 1661 & 58 & 81 & 83 & 55 & 8 & 1376 \\
\hline Chihuahua & 1558 & 639 & 60 & 128 & 731 & 63 & 1697 & 56 & 71 & 90 & 59 & 0 & 1421 \\
\hline Distrito Federal & 9650 & 5194 & 1004 & 978 & 2474 & 809 & 15526 & 245 & 1249 & 815 & 385 & 26 & 12806 \\
\hline Durango & 1161 & 594 & 78 & 116 & 373 & 82 & 1021 & 29 & 58 & 61 & 32 & 6 & 835 \\
\hline Guanajuato & 2083 & 755 & 60 & 165 & 1103 & 201 & 1574 & 48 & 110 & 89 & 49 & 16 & 1262 \\
\hline Guerrero & 2407 & 1464 & 82 & 215 & 646 & 96 & 2198 & 129 & 101 & 72 & 43 & 0 & 1853 \\
\hline Hidalgo & 1483 & 605 & 51 & 143 & 684 & 123 & 1366 & 13 & 85 & 70 & 23 & 1 & 1174 \\
\hline Jalisco & 4745 & 2026 & 270 & 275 & 2174 & 324 & 4241 & 135 & 311 & 156 & 101 & 28 & 3510 \\
\hline México & 5508 & 1827 & 188 & 1230 & 2263 & 579 & 8427 & 95 & 259 & 353 & 134 & 21 & 7565 \\
\hline Michoacán & 1812 & 1023 & 90 & 413 & 286 & 112 & 1789 & 128 & 87 & 28 & 32 & 3 & 1511 \\
\hline Morelos & 1118 & 617 & 68 & 162 & 271 & 78 & 885 & 13 & 53 & 68 & 33 & 1 & 717 \\
\hline$N$ ayarit & 770 & 500 & 57 & 44 & 169 & 29 & 447 & 12 & 21 & 30 & 21 & 1 & 362 \\
\hline N uevo León & 1706 & 794 & 53 & 413 & 446 & 128 & 1533 & 97 & 73 & 50 & 39 & 10 & 1264 \\
\hline 0 axaca & 1765 & 854 & 77 & 247 & 587 & 191 & 1480 & 21 & 51 & 68 & 37 & 12 & 1291 \\
\hline Puebla & 2063 & 809 & 96 & 667 & 491 & 91 & 1457 & 55 & 76 & 49 & 44 & 1 & 1232 \\
\hline Q uerétaro & 1129 & 403 & 71 & 65 & 590 & 45 & 880 & 24 & 52 & 38 & 30 & 2 & 734 \\
\hline Q uintana Roo & 681 & 259 & 4 & 49 & 369 & 36 & 543 & 24 & 19 & 43 & 24 & 0 & 433 \\
\hline San Luis Potosí & 1339 & 592 & 28 & 156 & 563 & 177 & 1345 & 27 & 68 & 41 & 25 & 0 & 1184 \\
\hline Sinaloa & 1422 & 670 & 30 & 269 & 453 & 133 & 1529 & 81 & 75 & 34 & 35 & 11 & 1293 \\
\hline Sonora & 1852 & 771 & 38 & 269 & 774 & 149 & 2147 & 123 & 133 & 52 & 52 & 2 & 1785 \\
\hline Tabasco & 2371 & 753 & 67 & 112 & 1439 & 127 & 2450 & 71 & 186 & 109 & 52 & 6 & 2026 \\
\hline Tamaulipas & 2103 & 1022 & 212 & 151 & 718 & 144 & 2317 & 85 & 121 & 96 & 74 & 8 & 1933 \\
\hline Tlaxcala & 678 & 348 & 15 & 126 & 189 & 33 & 483 & 8 & 27 & 32 & 16 & 0 & 400 \\
\hline Veracruz & 2826 & 1315 & 87 & 390 & 1034 & 128 & 2706 & 100 & 145 & 85 & 55 & 19 & 2302 \\
\hline Yucatán & 1114 & 285 & 38 & 201 & 590 & 89 & 933 & 48 & 50 & 18 & 20 & 3 & 794 \\
\hline Zacatecas & 793 & 450 & 11 & 110 & 222 & 35 & 739 & 32 & 25 & 26 & 15 & 7 & 634 \\
\hline Institutos nacionales & 3920 & 2437 & 682 & 176 & 625 & 455 & 7449 & 341 & 276 & 447 & 176 & 24 & 6185 \\
\hline
\end{tabular}

Fuente: Secretaría de Salud (SSA), Subsecretaría de Prevención y Control de Enfermedades, D irección G eneral de Estadística e Informática (D GEI), Sistema de Información en Salud para Población A bierta (SISPA), Registro N acional de Infraestructura para la Salud (REN IS) 


\section{Recursos humanos en las unidades médicas. Secretaría de Salud, México, 1999}

\begin{tabular}{|c|c|c|c|c|c|c|c|c|c|c|c|c|}
\hline \multirow[b]{2}{*}{ Entidad federativa } & \multicolumn{6}{|c|}{ Número } & \multicolumn{6}{|c|}{ Porcentaje } \\
\hline & Total & $\begin{array}{l}\text { Mé- } \\
\text { dicos }\end{array}$ & $\begin{array}{l}\text { Enfer- } \\
\text { meras }\end{array}$ & $\begin{array}{c}\text { Otros } \\
\text { paramédicos }\end{array}$ & $\begin{array}{l}\text { Adminis- } \\
\text { trativo }\end{array}$ & $\begin{array}{c}\text { Otro } \\
\text { personal }\end{array}$ & Total & $\begin{array}{l}\text { Mé- } \\
\text { dicos }\end{array}$ & $\begin{array}{l}\text { Enfer- } \\
\text { meras }\end{array}$ & $\begin{array}{c}\text { Otros } \\
\text { paramédicos }\end{array}$ & $\begin{array}{l}\text { Adminis- } \\
\text { trativo }\end{array}$ & $\begin{array}{c}\text { Otro } \\
\text { personal }\end{array}$ \\
\hline Estados Unidos Mexicanos & 193397 & 50309 & 69678 & 18543 & 38839 & 16028 & 100.00 & 26.01 & 36.03 & 9.59 & 20.08 & 8.29 \\
\hline A guascalientes & 2839 & 838 & 1002 & 186 & 518 & 295 & 100.00 & 29.52 & 35.29 & 6.55 & 18.25 & 10.39 \\
\hline Baja C alifornia & 2887 & 771 & 1195 & 189 & 580 & 152 & 100.00 & 26.71 & 41.39 & 6.55 & 20.09 & 5.26 \\
\hline Baja C alifornia Sur & 1462 & 357 & 483 & 143 & 314 & 165 & 100.00 & 24.42 & 33.04 & 9.78 & 21.48 & 11.29 \\
\hline Campeche & 1780 & 513 & 650 & 132 & 290 & 195 & 100.00 & 28.82 & 36.52 & 7.42 & 16.29 & 10.96 \\
\hline Coahuila & 3105 & 837 & 1284 & 249 & 536 & 199 & 100.00 & 26.96 & 41.35 & 8.02 & 17.26 & 6.41 \\
\hline Colima & 1374 & 416 & 514 & 114 & 248 & 82 & 100.00 & 30.28 & 37.41 & 8.30 & 18.05 & 5.97 \\
\hline Chiapas & 5100 & 1403 & 2036 & 405 & 727 & 529 & 100.00 & 27.51 & 39.92 & 7.94 & 14.25 & 10.37 \\
\hline Chihuahua & 4384 & 1066 & 1621 & 380 & 752 & 565 & 100.00 & 24.32 & 36.98 & 8.67 & 17.15 & 12.89 \\
\hline Distrito Federal & 33482 & 7497 & 10459 & 3812 & 9168 & 2546 & 100.00 & 22.39 & 31.24 & 11.39 & 27.38 & 7.60 \\
\hline Durango & 3064 & 800 & 1243 & 246 & 541 & 234 & 100.00 & 26.11 & 40.57 & 8.03 & 17.66 & 7.64 \\
\hline Guanajuato & 5669 & 1811 & 2284 & 432 & 704 & 438 & 100.00 & 31.95 & 40.29 & 7.62 & 12.42 & 7.73 \\
\hline Guerrero & 6731 & 2030 & 2503 & 746 & 1038 & 414 & 100.00 & 30.16 & 37.19 & 11.08 & 15.42 & 6.15 \\
\hline Hidalgo & 4234 & 1262 & 1606 & 466 & 630 & 270 & 100.00 & 29.81 & 37.93 & 11.01 & 14.88 & 6.38 \\
\hline Jalisco & 13302 & 3992 & 5069 & 977 & 2668 & 596 & 100.00 & 30.01 & 38.11 & 7.34 & 20.06 & 4.48 \\
\hline México & 18997 & 4483 & 6087 & 3232 & 3400 & 1795 & 100.00 & 23.60 & 32.04 & 17.01 & 17.90 & 9.45 \\
\hline Michoacán & 5382 & 1669 & 1924 & 346 & 978 & 465 & 100.00 & 31.01 & 35.75 & 6.43 & 18.17 & 8.64 \\
\hline Morelos & 2938 & 857 & 1196 & 208 & 423 & 254 & 100.00 & 29.17 & 40.71 & 7.08 & 14.40 & 8.65 \\
\hline $\mathrm{N}$ ayarit & 1843 & 597 & 799 & 93 & 240 & 114 & 100.00 & 32.39 & 43.35 & 5.05 & 13.02 & 6.19 \\
\hline Nuevo León & 4743 & 1376 & 1834 & 366 & 709 & 458 & 100.00 & 29.01 & 38.67 & 7.72 & 14.95 & 9.66 \\
\hline 0 axaca & 4909 & 1473 & 1956 & 330 & 760 & 390 & 100.00 & 30.01 & 39.85 & 6.72 & 15.48 & 7.94 \\
\hline Puebla & 5320 & 1709 & 2154 & 313 & 680 & 464 & 100.00 & 32.12 & 40.49 & 5.88 & 12.78 & 8.72 \\
\hline Q uerétaro & 2921 & 867 & 1174 & 177 & 466 & 237 & 100.00 & 29.68 & 40.19 & 6.06 & 15.95 & 8.11 \\
\hline Q uintana Roo & 1801 & 541 & 717 & 127 & 298 & 118 & 100.00 & 30.04 & 39.81 & 7.05 & 16.55 & 6.55 \\
\hline San Luis Potosí & 3840 & 979 & 1516 & 252 & 597 & 496 & 100.00 & 25.49 & 39.48 & 6.56 & 15.55 & 12.92 \\
\hline Sinaloa & 4076 & 992 & 1555 & 297 & 593 & 639 & 100.00 & 24.34 & 38.15 & 7.29 & 14.55 & 15.68 \\
\hline Sonora & 5337 & 1189 & 2001 & 472 & 1155 & 520 & 100.00 & 22.28 & 37.49 & 8.84 & 21.64 & 9.74 \\
\hline Tabasco & 6814 & 1866 & 2498 & 562 & 1249 & 639 & 100.00 & 27.38 & 36.66 & 8.25 & 18.33 & 9.38 \\
\hline Tamaulipas & 5991 & 1427 & 2247 & 489 & 1371 & 457 & 100.00 & 23.82 & 37.51 & 8.16 & 22.88 & 7.63 \\
\hline Tlaxcala & 1766 & 572 & 711 & 102 & 323 & 58 & 100.00 & 32.39 & 40.26 & 5.78 & 18.29 & 3.28 \\
\hline Veracruz & 8099 & 2439 & 2954 & 589 & 1521 & 596 & 100.00 & 30.11 & 36.47 & 7.27 & 18.78 & 7.36 \\
\hline Yucatán & 2933 & 797 & 1203 & 192 & 461 & 280 & 100.00 & 27.17 & 41.02 & 6.55 & 15.72 & 9.55 \\
\hline Zacatecas & 2146 & 579 & 828 & 137 & 311 & 291 & 100.00 & 26.98 & 38.58 & 6.38 & 14.49 & 13.56 \\
\hline Institutos nacionales & 14128 & 2304 & 4375 & 1782 & 4590 & 1077 & 100.00 & 16.31 & 30.97 & 12.61 & 32.49 & 7.62 \\
\hline
\end{tabular}

Fuente: Secretaría de Salud (SSA), Subsecretaría de Prevención y Control de Enfermedades, Dirección General de Estadística e Informática (D G El), Sistema de Información en Salud para Población Abierta (SISPA), Registro N acional de Infraestructura para la Salud (REN IS) 


\section{Cuadro V \\ Recursos humanos por tipo de unidad. Secretaría de Salud, México, 1999}

\begin{tabular}{|c|c|c|c|c|c|c|c|c|c|c|c|c|c|}
\hline \multirow[b]{2}{*}{ Tipo de unidad } & \multirow[b]{2}{*}{$\begin{array}{c}\text { M édicos } \\
\text { en contacto }\end{array}$} & \multirow[b]{2}{*}{ Otra } & \multicolumn{7}{|c|}{ M édicos en contacto con el paciente } & \multicolumn{4}{|c|}{ En formación } \\
\hline & & & $\begin{array}{c}\text { General } \\
\text { y familiar }\end{array}$ & $\begin{array}{c}\text { Pedia- } \\
\text { tra }\end{array}$ & $\begin{array}{l}\text { Gineco } \\
\text { obstétra }\end{array}$ & $\begin{array}{l}\text { Ciru- } \\
\text { jano }\end{array}$ & $\begin{array}{l}\text { Inter- } \\
\text { nista }\end{array}$ & $\begin{array}{c}\text { Otra } \\
\text { especialidad }\end{array}$ & $\begin{array}{l}\text { Odon- } \\
\text { tólogo }\end{array}$ & $\begin{array}{l}\text { Pasante } \\
\text { medicina }\end{array}$ & $\begin{array}{c}\text { Pasante } \\
\text { odontólogo }\end{array}$ & $\begin{array}{l}\text { Internos de } \\
\text { pregrado }\end{array}$ & $\begin{array}{l}\text { Resi- } \\
\text { dente }\end{array}$ \\
\hline stados Unidos Mexicanos & 46057 & 4252 & 13239 & 2200 & 2073 & 1985 & 1088 & 6769 & 3238 & 5840 & 1609 & 3254 & 4762 \\
\hline nidad rural & 9791 & 379 & 3764 & 4 & 3 & 1 & 1 & 3 & 528 & 5055 & 432 & 0 & 0 \\
\hline Unidad móvil & 1888 & 0 & 1051 & 0 & 0 & 0 & 0 & 0 & 391 & 361 & 85 & 0 & 0 \\
\hline Unidad urbana & 7000 & 932 & 4491 & 20 & 29 & 13 & 10 & 67 & 1348 & 333 & 679 & 2 & 8 \\
\hline Clínica de especialidad & 688 & 104 & 90 & 20 & 20 & 24 & 5 & 241 & 85 & 0 & 141 & 17 & 45 \\
\hline C. de S. con hospitalización & 1640 & 141 & 963 & 58 & 92 & 91 & 16 & 113 & 145 & 79 & 83 & 0 & 0 \\
\hline Hospital integral & 420 & 46 & 226 & 23 & 27 & 33 & 5 & 44 & 32 & 11 & 16 & 3 & 0 \\
\hline Hospital general & 16100 & 1355 & 2047 & 1203 & 1416 & 1443 & 897 & 3546 & 417 & 1 & 134 & 2611 & 2385 \\
\hline Hospital especializado & 5954 & 694 & 413 & 657 & 446 & 308 & 118 & 1928 & 221 & 0 & 12 & 618 & 1233 \\
\hline Hospital psiquiátrico & 737 & 136 & 186 & 5 & 1 & 2 & 16 & 318 & 35 & 0 & 27 & 3 & 144 \\
\hline Institutos nacionale & 1839 & 465 & 8 & 210 & 39 & 70 & 20 & 509 & 36 & 0 & 0 & 0 & 947 \\
\hline
\end{tabular}

Fuente: Secretaría de Salud (SSA), Subsecretaría de Prevención y Control de Enfermedades, Dirección General de Estadística e Informática (D G EI), Sistema de Información en Salud para Población A bierta (SISPA), Registro N acional de Infraestructura para la Salud (REN IS)

\section{Recursos humanos por tipo de unidad. Secretaría de Salud, México, 1999}

\begin{tabular}{|c|c|c|c|c|c|c|c|c|c|c|c|}
\hline \multirow[t]{2}{*}{ Tipo de unidad } & \multirow[t]{2}{*}{ Total } & \multicolumn{2}{|c|}{ Médicos } & \multicolumn{2}{|c|}{ Enfermeras } & \multicolumn{2}{|c|}{ Otros paramédicos } & \multicolumn{2}{|c|}{ Administrativo } & \multicolumn{2}{|c|}{ Otro personal } \\
\hline & & $n$ & $\%$ & $\mathrm{n}$ & $\%$ & $n$ & $\%$ & $n$ & $\%$ & $\mathrm{n}$ & $\%$ \\
\hline Estados Unidos Mexicanos & 193397 & 50309 & 26.01 & 69678 & 36.03 & 18543 & 9.59 & 38839 & 20.08 & 16028 & 8.29 \\
\hline Unidad rural & 24529 & 10170 & 41.46 & 9866 & 40.22 & 1795 & 7.32 & 1304 & 5.32 & 1394 & 5.68 \\
\hline Unidad móvil & 4646 & 1888 & 40.64 & 1397 & 30.07 & 182 & 3.92 & 1 & 0.02 & 1178 & 25.36 \\
\hline Unidad urbana & 28331 & 7932 & 28.00 & 8869 & 31.30 & 3462 & 12.22 & 5239 & 18.49 & 2829 & 9.99 \\
\hline Clínica de especialidad & 2623 & 792 & 30.19 & 475 & 18.11 & 509 & 19.41 & 691 & 26.34 & 156 & 5.95 \\
\hline C. de S. con hospitalización & 6112 & 1781 & 29.14 & 2484 & 40.64 & 422 & 6.90 & 911 & 14.91 & 514 & 8.41 \\
\hline Hospital integral & 1829 & 466 & 25.48 & 721 & 39.42 & 131 & 7.16 & 312 & 17.06 & 199 & 10.88 \\
\hline Hospital general & 73219 & 17455 & 23.84 & 28056 & 38.32 & 6391 & 8.73 & 15746 & 21.51 & 5571 & 7.61 \\
\hline Hospital especializado & 31688 & 6648 & 20.98 & 11031 & 34.81 & 3097 & 9.77 & 8344 & 26.33 & 2568 & 8.10 \\
\hline Hospital psiquiátrico & 6292 & 873 & 13.87 & 2404 & 38.21 & 772 & 12.27 & 1701 & 27.03 & 542 & 8.61 \\
\hline Institutos nacionales & 14128 & 2304 & 16.31 & 4375 & 30.97 & 1782 & 12.61 & 4590 & 32.49 & 1077 & 7.62 \\
\hline
\end{tabular}

Fuente: Secretaría de Salud (SSA), Subsecretaría de Prevención y Control de Enfermedades, D irección General de Estadística e Informática (D G El), Sistema de Información en Salud para Población A bierta (SISPA), Registro N acional de Infraestructura para la Salud (REN IS) 
Cuadro VII

Indicadores de recursos. Secretaría de Salud, México, 1999

\begin{tabular}{|c|c|c|c|c|c|c|c|}
\hline \multirow[b]{2}{*}{ Entidad federativa } & \multicolumn{4}{|c|}{ Por 100000 habitantes de población potencial* } & \multirow{2}{*}{$\begin{array}{l}\text { Porcentaje de } \\
\text { médicos en } \\
\text { formación }\end{array}$} & \multirow{2}{*}{$\begin{array}{c}\text { Relación } \\
\text { generales/ } \\
\text { especialistas }\end{array}$} & \multirow[b]{2}{*}{$\begin{array}{l}\text { Enfermeras } \\
\text { por médico }\end{array}$} \\
\hline & $\begin{array}{l}\text { Camas } \\
\text { censables }\end{array}$ & $\begin{array}{l}\text { Consul- } \\
\text { torios }\end{array}$ & Médicos & Enfermeras & & & \\
\hline Estados Unidos Mexicanos & 78.53 & 55.79 & 126.42 & 175.09 & 33.58 & 0.94 & 1.39 \\
\hline Aguascalientes & 93.48 & 59.07 & 198.81 & 237.72 & 35.09 & 0.48 & 1.20 \\
\hline Baja California & 57.03 & 47.97 & 120.47 & 186.72 & 42.49 & 0.66 & 1.55 \\
\hline Baja California Sur & 77.74 & 49.47 & 126.15 & 170.67 & 32.15 & 0.62 & 1.35 \\
\hline Campeche & 82.44 & 65.81 & 120.14 & 152.22 & 25.31 & 1.08 & 1.27 \\
\hline Coahuila & 57.80 & 53.33 & 104.04 & 159.60 & 27.36 & 0.81 & 1.53 \\
\hline Colima & 51.41 & 56.01 & 106.39 & 131.46 & 36.22 & 0.78 & 1.24 \\
\hline Chiapas & 44.89 & 49.29 & 86.98 & 126.22 & 19.13 & 1.47 & 1.45 \\
\hline Chihuahua & 106.62 & 58.91 & 135.62 & 206.23 & 22.59 & 1.01 & 1.52 \\
\hline Distrito Federal $^{\ddagger}$ & 179.91 & 88.26 & 272.70 & 412.74 & 28.81 & 0.40 & 1.51 \\
\hline Durango & 77.17 & 55.59 & 125.98 & 195.75 & 26.77 & 0.95 & 1.55 \\
\hline Guanajuato & 58.72 & 52.78 & 107.64 & 135.75 & 35.32 & 2.15 & 1.26 \\
\hline Guerrero & 33.93 & 51.27 & 82.99 & 102.33 & 25.49 & 2.24 & 1.23 \\
\hline Hidalgo & 64.52 & 55.38 & 113.08 & 143.91 & 37.35 & 2.46 & 1.27 \\
\hline Jalisco & 97.16 & 56.96 & 157.48 & 199.96 & 49.07 & 0.62 & 1.27 \\
\hline México & 91.45 & 48.87 & 95.68 & 129.91 & 38.73 & 1.22 & 1.36 \\
\hline Michoacán & 58.70 & 45.82 & 110.82 & 127.76 & 42.24 & 1.02 & 1.15 \\
\hline Morelos & 41.82 & 52.52 & 105.41 & 147.11 & 29.05 & 0.62 & 1.40 \\
\hline$N$ ayarit & 57.36 & 55.49 & 111.17 & 148.79 & 40.96 & 1.26 & 1.34 \\
\hline Nuevo León & 34.39 & 46.49 & 75.71 & 100.91 & 41.80 & 1.32 & 1.33 \\
\hline 0 axaca & 51.58 & 47.52 & 98.17 & 130.36 & 38.14 & 1.99 & 1.33 \\
\hline Puebla & 66.95 & 51.56 & 95.26 & 120.07 & 43.95 & 1.54 & 1.26 \\
\hline Q uerétaro & 50.27 & 45.16 & 116.53 & 157.80 & 40.86 & 0.82 & 1.35 \\
\hline Q uintana Roo & 50.41 & 56.71 & 109.96 & 145.73 & 20.51 & 1.66 & 1.33 \\
\hline San Luis Potosí & 67.38 & 42.55 & 110.50 & 171.11 & 36.12 & 1.04 & 1.55 \\
\hline Sinaloa & 65.00 & 55.38 & 134.33 & 210.56 & 29.47 & 0.78 & 1.57 \\
\hline Sonora & 130.27 & 71.51 & 151.56 & 255.07 & 39.83 & 0.58 & 1.68 \\
\hline Tabasco & 56.59 & 56.87 & 128.78 & 172.39 & 25.94 & 1.46 & 1.34 \\
\hline Tamaulipas & 136.58 & 65.73 & 173.71 & 273.52 & 28.06 & 0.73 & 1.57 \\
\hline Tlaxcala & 43.51 & 52.97 & 98.37 & 122.27 & 34.55 & 1.38 & 1.24 \\
\hline Veracruz & 78.00 & 57.14 & 116.92 & 141.61 & 23.53 & 1.41 & 1.21 \\
\hline Yucatán & 81.54 & 51.96 & 133.17 & 201.00 & 31.51 & 0.88 & 1.51 \\
\hline Zacatecas & 49.19 & 45.26 & 98.89 & 141.42 & 31.14 & 0.97 & 1.43 \\
\hline
\end{tabular}

* Esta población se obtiene a partir del número de consultorios que tiene cada entidad por tipo de unidad

₹ Incluye institutos nacionales

Fuente: Secretaría de Salud (SSA), Subsecretaría de Prevención y C ontrol de Enfermedades, D irección General de Estadística e Informática (D G El), Sistema de Información en Salud para Población Abierta (SISPA), Registro N acional de Infraestructura para la Salud (REN IS) 